\title{
The energy-dependent black-disk fraction in proton-proton scattering
}

\author{
Dieter Schildknecht \\ Fakultät für Physik, Universität Bielefeld, D-33501 Bielefeld, Germany \\ and Max-Planck-Institut für Physik (Werner-Heisenberg-Institut), \\ Föhringer Ring 6, D-80805 München, Germany
}

(Received 14 June 2018; published 12 November 2018)

\begin{abstract}
Recent work by Block et al. on the energy-invariant edge in proton-proton scattering is interpreted by quantitatively introducing an energy-dependent black-disk fraction of the proton-proton interaction.
\end{abstract}

DOI: 10.1103/PhysRevD.98.094009

In the context of the investigations on the empirical evidence for the approach of the proton-proton-scattering cross section to a black-disk limit at very high energies, the variable

$$
t=\frac{\sigma^{\mathrm{TOT}}\left(W^{2}\right)-2 \sigma^{\mathrm{EL}}\left(W^{2}\right)}{\sqrt{\frac{\pi \sigma^{\mathrm{TOT}}\left(W^{2}\right)}{2}}}
$$

was introduced [1]; compare also Refs. [2-4].

The numerical values of $t$ can be determined from the experimental results for the total $p p$ cross section, $\sigma^{\mathrm{TOT}}\left(W^{2}\right)$, and the elastic one, $\sigma^{\mathrm{EL}}\left(W^{2}\right)$, measured at the center-of-mass energy $W$. In an impact-parameter description of $p p$ scattering, $t$ stands for the width of the edge of the scattering region in impact-parameter space [1]. Employing $t$, in the present paper, we wish to explicitly elaborate on how the variable $t$ specifies the fraction of the total cross section due to black-disk scattering as a function of the energy $W$.

From the experimental results on $\sigma^{\mathrm{TOT}}$ and $\sigma^{\mathrm{EL}}$, technically by evaluating precise fits $[1,2,3,5]$ to the experimental data for $\sigma^{\mathrm{TOT}}, \sigma^{\mathrm{EL}}$, and the inelastic cross section $\sigma^{\mathrm{INEL}}\left(W^{2}\right)$, it was found that $t$ in (1) is independent of the energy $W$ :

$$
t=\text { const } \simeq 1.1 \mathrm{fm} .
$$

From the extrapolation of the fits to energies far beyond the presently accessible ones, the remarkable constancy of $t \simeq$ $1.1 \mathrm{fm}$ in (2) was found to remain valid in the huge range of $W \simeq 10 \mathrm{GeV}$ to $W \simeq 10^{13} \mathrm{GeV}[1]$.

Published by the American Physical Society under the terms of the Creative Commons Attribution 4.0 International license. Further distribution of this work must maintain attribution to the author(s) and the published article's title, journal citation, and DOI. Funded by SCOAP ${ }^{3}$.
The significance of the empirical observation (2) is best illuminated by rewriting (1) as

$$
\sigma^{\mathrm{EL}}\left(W^{2}\right)=\frac{1}{2} \sigma^{\mathrm{TOT}}\left(W^{2}\right)\left(1-\frac{t}{\sqrt{\frac{2 \sigma^{\mathrm{TOT}}\left(W^{2}\right)}{\pi}}}\right) .
$$

Relation (3) is seen to correspond to the transition from two $W$-dependent observables $\left(\sigma^{\mathrm{TOT}}\left(W^{2}\right), \sigma^{\mathrm{EL}}\left(W^{2}\right)\right)$ to the two observables $\left(\sigma^{\mathrm{TOT}}\left(W^{2}\right), t \simeq\right.$ const $\left.\cong 1.1 \mathrm{fm}\right)$. A measured value of the single quantity $\sigma^{\mathrm{TOT}}\left(W^{2}\right)$ at the energy $W$, together with the constant $t \cong 1.1 \mathrm{fm}$, determines the elastic cross section in (3), as well as the inelastic one,

$$
\sigma^{\mathrm{INEL}}\left(W^{2}\right)=\frac{1}{2} \sigma^{\mathrm{TOT}}\left(W^{2}\right)\left(1+\frac{t}{\sqrt{\frac{2 \sigma^{\mathrm{TOT}}\left(W^{2}\right)}{\pi}}}\right) .
$$

Due to the slow growth of $\sigma^{\mathrm{TOT}}\left(W^{2}\right) \simeq c_{0}+$ $c_{2} \ln ^{2}\left(W^{2} / 2 m_{p}^{2}\right)$, where $c_{0} \simeq 24 \mathrm{mb}=2.4 \mathrm{fm}^{2}$ and $c_{2} \simeq$ $0.23 \mathrm{mb}=0.023 \mathrm{fm}^{2}$ [3], for $W \rightarrow \infty$, the black-disk limit

$$
\lim _{W \rightarrow \infty} \frac{\sigma^{\mathrm{EL}}\left(W^{2}\right)}{\sigma^{\mathrm{TOT}}\left(W^{2}\right)}=\frac{1}{2}
$$

in (3) is reached only at energies far beyond the ones available in the laboratory. Even for the very high energies explored at the Large Hadron Collider (LHC) and by the Auger [6] and HiRes collaborations [7], one finds [3] values of $\sigma^{\mathrm{EL}} / \sigma^{\mathrm{TOT}}$ of 0.28 (at $14 \mathrm{TeV}$ ) and 0.30 (at $57 \mathrm{TeV}$ ) still significantly below $\sigma^{\mathrm{EL}} / \sigma^{\mathrm{TOT}}=0.50$ in (5).

At finite energy $W$, the pair of observables $\left[\sigma^{\mathrm{TOT}}\left(W^{2}\right)\right.$, $t \simeq$ const $\simeq 1.1 \mathrm{fm}]$ according to (1) determines the normalized deviation of the total cross section from the black-disk limit (5),

$$
\frac{\sigma^{\mathrm{TOT}}\left(W^{2}\right)-2 \sigma^{\mathrm{EL}}\left(W^{2}\right)}{\sigma^{\mathrm{TOT}}\left(W^{2}\right)}=\frac{t}{\sqrt{\frac{2 \sigma^{\mathrm{TOT}}\left(W^{2}\right)}{\pi}}},
$$

as well as the normalized difference between the inelastic and the elastic cross section, given by 


$$
\frac{\sigma^{\mathrm{INEL}}\left(W^{2}\right)-\sigma^{\mathrm{EL}}\left(W^{2}\right)}{\sigma^{\mathrm{TOT}}\left(W^{2}\right)}=\frac{t}{\sqrt{\frac{2 \sigma^{\mathrm{TOT}}\left(W^{2}\right)}{\pi}}},
$$

where $\sigma^{\mathrm{TOT}}\left(W^{2}\right)=\sigma^{\mathrm{EL}}\left(W^{2}\right)+\sigma^{\mathrm{INEL}}\left(W^{2}\right)$ is used in the transition from (6) to (7). The limit of $W \rightarrow \infty$ in (6) takes us back to (5).

It is useful to define a black-disk total cross section, $\sigma_{\text {Black }}^{\text {TOT }}\left(W^{2}\right)$, associated with a given value of the elastic cross section via

$$
\sigma_{\text {Black }}^{\mathrm{TOT}}\left(W^{2}\right)=2 \sigma^{\mathrm{EL}}\left(W^{2}\right) .
$$

Inserting (8) into (6) yields

$$
\sigma^{\mathrm{TOT}}\left(W^{2}\right)=\sigma_{\text {Black }}^{\mathrm{TOT}}\left(W^{2}\right)+t \sqrt{\frac{\pi}{2} \sigma^{\mathrm{TOT}}\left(W^{2}\right)} .
$$

The total cross section is accordingly recognized as the sum of a contribution that is associated with a black disk according to (8), and a contribution that is proportional to the constant $t$. The fraction of the total cross section originating from the black-disk interaction according to (9) is given by

$$
\frac{\sigma_{\mathrm{Black}}^{\mathrm{TOT}}\left(W^{2}\right)}{\sigma^{\mathrm{TOT}}\left(W^{2}\right)}=1-\frac{t}{\sqrt{\frac{2 \sigma^{\mathrm{TOT}}\left(W^{2}\right)}{\pi}}}=\left\{\begin{array}{ll}
1 & \text { for } \sqrt{\frac{2 \sigma^{\mathrm{TOT}}\left(W^{2}\right)}{\pi}} \gg t, \\
\frac{1}{2} & \text { for } \sqrt{\frac{2 \sigma^{\mathrm{TOT}}\left(W^{2}\right)}{\pi}}=2 t
\end{array} .\right.
$$

In (10), besides the asymptotic value of unity (5), we have given the numerical value of the ratio $\sigma_{\text {Black }}^{\text {TOT }}\left(W^{2}\right) /$ $\sigma^{\mathrm{TOT}}\left(W^{2}\right)=1 / 2$ that is obtained for $\sigma^{\mathrm{TOT}}=2 \pi t^{2}$. With the empirical value of $t=1.1 \mathrm{fm}$, this value of $\sigma^{\mathrm{TOT}}\left(W^{2}\right)=$ $2 \pi t^{2}$ coincides with the measurements of $\sigma^{\mathrm{TOT}}\left(W^{2}\right)$ at the energy of approximately $W \cong 2 \mathrm{TeV}[1,2,3]$. From (10), at $W \cong 2 \mathrm{TeV}$, the black disk defined by (8) contributes about one half of the sum on the right-hand side in (9). Or, equivalently, the cross section of a black disk determined by a given value of $\sigma^{\mathrm{EL}}\left(W^{2}\right)$ according to (8), for $W=2 \mathrm{TeV}$, is only half as large as the empirical cross section according to (9).

Additional insight into the significance of the representation (9) is obtained by introducing radii for the cross sections $\sigma^{\mathrm{TOT}}\left(W^{2}\right)$ and $\sigma_{\text {Black }}^{\mathrm{TOT}}\left(W^{2}\right)$, respectively:

$$
\sigma^{\mathrm{TOT}}\left(W^{2}\right)=2 \pi R_{\mathrm{TOT}}^{2}\left(W^{2}\right),
$$

as well as

$$
\sigma_{\text {Black }}^{\mathrm{TOT}}\left(W^{2}\right)=2 \sigma^{\mathrm{EL}}\left(W^{2}\right)=2 \pi R_{\text {Black }}^{2}\left(W^{2}\right) .
$$

In distinction from the black-disk radius in (12), $R_{\text {Black }}\left(W^{2}\right)$, the radius $R_{\mathrm{TOT}}\left(W^{2}\right)$ introduced in (11) must be considered as an effective radius. It approaches the radius of a black disk, $R_{\mathrm{TOT}}\left(W^{2}\right) \rightarrow R_{\mathrm{Black}}\left(W^{2}\right)$, in the asymptotic limit of $W \rightarrow \infty$, where $\sigma^{\mathrm{TOT}}\left(W^{2}\right) \rightarrow$ $\sigma_{\text {Black }}^{\text {TOT }}\left(W^{2}\right)$; compare (5) and (8).

With (11), the cross section (9) is represented by

$$
\sigma^{\mathrm{TOT}}\left(W^{2}\right)=\sigma_{\mathrm{Black}}^{\mathrm{TOT}}\left(W^{2}\right)+\pi t R_{\mathrm{TOT}}\left(W^{2}\right)
$$

Note that (13) is an empirical relation that only rests on the defining Eqs. (1), (11), and (12) in terms of the measurable cross sections $\sigma^{\mathrm{TOT}}\left(W^{2}\right)=\sigma^{\mathrm{EL}}\left(W^{2}\right)+$ $\sigma^{\mathrm{INEL}}\left(W^{2}\right)$ and $\sigma^{\mathrm{EL}}\left(W^{2}\right)$. Note also that $\pi t R_{\mathrm{TOT}}\left(W^{2}\right)=$ $\pi t \sqrt{\sigma^{\text {TOT }}\left(W^{2}\right) / 2 \pi}$ is a measurable quantity. The additive contribution $\pi t R_{\mathrm{TOT}}\left(W^{2}\right)$ in (13), properly called the "edge" [1], is recognized as a contribution to $\sigma^{\mathrm{TOT}}\left(W^{2}\right)$ that is proportional to the area $2 \pi t R_{\mathrm{TOT}}\left(W^{2}\right)$ of a ring of constant thickness (or width) $t=$ const centered around the radius $R_{\mathrm{TOT}}\left(W^{2}\right)$. Multiplication by a factor $2 \times 1 / 4$, where the factor 2 takes blackness into account, and $1 / 4$ is due to decreasing absorption [1,2], leads to $\pi t R_{\mathrm{TOT}}\left(W^{2}\right)$ in (13). Returning to $\sigma^{\mathrm{INEL}}\left(W^{2}\right)$ and $\sigma^{\mathrm{EL}}\left(W^{2}\right)$ in (13), we see that the edge contribution yields the excess of $\sigma^{\mathrm{INEL}}\left(W^{2}\right)$ over $\sigma^{\mathrm{EL}}\left(W^{2}\right)$,

$$
\sigma^{\mathrm{INEL}}\left(W^{2}\right)-\sigma^{\mathrm{EL}}\left(W^{2}\right)=\pi t R_{\mathrm{TOT}}\left(W^{2}\right) .
$$

The ratio (10) of $\sigma_{\text {Black }}^{\text {TOT }}\left(W^{2}\right)$ to $\sigma^{\mathrm{TOT}}\left(W^{2}\right)$, with (11) to (13), at finite energy $W$ deviates from the asymptotic value of unity by the normalized edge contribution. Explicitly,

$$
\frac{\sigma_{\mathrm{Black}}^{\mathrm{TOT}}\left(W^{2}\right)}{\sigma^{\mathrm{TOT}}\left(W^{2}\right)}=1-\frac{t}{2 R_{\mathrm{TOT}}\left(W^{2}\right)}
$$

as well as

$$
\begin{aligned}
\frac{R_{\mathrm{Black}}\left(W^{2}\right)}{R_{\mathrm{TOT}}\left(W^{2}\right)} & =\sqrt{1-\frac{t}{2 R_{\mathrm{TOT}}\left(W^{2}\right)}} \\
& =\left\{\begin{array}{l}
1 \text { for } R_{\mathrm{TOT}}\left(W^{2}\right) \gg t, \\
\frac{1}{\sqrt{2}} \cong 0.71 \text { for } R_{\mathrm{TOT}}\left(W^{2}\right)=t .
\end{array}\right.
\end{aligned}
$$

At energies of approximately $W \cong 2 \mathrm{TeV}$, a region defined by an interaction radius of about $71 \%$ of the full interaction radius is associated with a black-disk interaction; the remaining part is due to the edge. Explicitly,

$$
\begin{aligned}
\frac{\sigma^{\mathrm{TOT}}\left(W^{2}\right)-\sigma_{\mathrm{Black}}^{\mathrm{TOT}}\left(W^{2}\right)}{\sigma^{\mathrm{TOT}}\left(W^{2}\right)} & =\frac{t}{2 R_{\mathrm{TOT}}\left(W^{2}\right)} \\
& = \begin{cases}0, & \text { for } R_{\mathrm{TOT}}\left(W^{2}\right) \gg t, \\
\frac{1}{2}, & \text { for } R_{\mathrm{TOT}}\left(W^{2}\right)=t,\end{cases}
\end{aligned}
$$



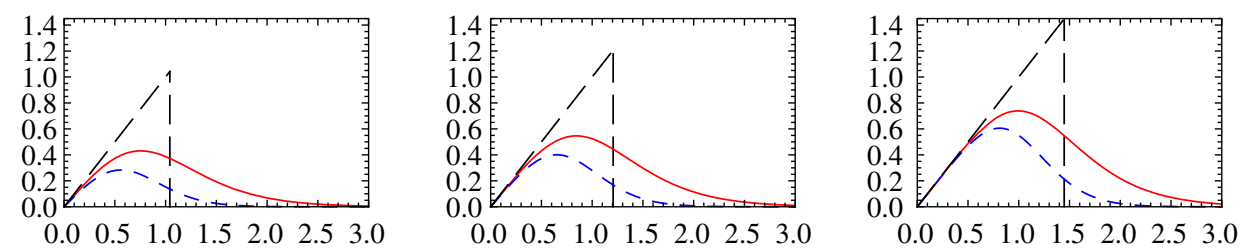

FIG. 1. The figures show amplitudes as a function of the impact parameter $b$ for energies of $W=1,5$, and 50 TeV. Integration over $b$ and multiplication by $4 \pi$ yields the total cross section $\sigma^{\mathrm{TOT}}\left(W^{2}\right)$ (solid red curve), the cross section $\sigma_{\text {Black }}^{\mathrm{TOT}}\left(W^{2}\right)=2 \sigma^{\mathrm{EL}}\left(W^{2}\right)(\operatorname{short}$ dashed blue curve), and the cross section of a black disk of radius $R_{\mathrm{TOT}}\left(W^{2}\right)=\sqrt{\sigma^{\mathrm{TOT}}\left(W^{2}\right) / 2 \pi}$ (long-dashed black curve). The vertical and horizontal scales give the amplitude and $b$ in $\mathrm{fm}$. The integral over the amplitude corresponding to $\sigma^{\mathrm{TOT}}\left(W^{2}\right)$ is equal to the integral over the black-disk amplitude associated with radius $R_{\mathrm{TOT}}\left(W^{2}\right)$. The region between the $\sigma^{\mathrm{TOT}}\left(W^{2}\right)$ and $\sigma_{\text {Black }}^{\mathrm{TOT}}\left(W^{2}\right)$ amplitudes is due to the edge. This figure is based on the eikonal fits in Ref. [2], and it was prepared by Phuoc Ha for the author of the present work.

as well as

$$
\begin{aligned}
\frac{R_{\mathrm{TOT}}\left(W^{2}\right)-R_{\mathrm{Black}}\left(W^{2}\right)}{R_{\mathrm{TOT}}\left(W^{2}\right)} & =1-\sqrt{1-\frac{t}{2 R_{\mathrm{TOT}}\left(W^{2}\right)}} \\
& =\left\{\begin{array}{l}
0 \text { for } R_{\mathrm{TOT}}\left(W^{2}\right) \gg t \\
0.29 \text { for } R_{\mathrm{TOT}}\left(W^{2}\right)=t
\end{array} .\right.
\end{aligned}
$$

For $W \rightarrow \infty$, the relative contribution from the edge to (9), according to (17) and (18) tends to zero; the asymptotic limit (3) is reached.

To gain further insight into the interplay between the black-disk part of the total cross section and the edge in (9) and (13), we go back to the impact-parameter representation, which actually was the starting point when quantifying the concept of a smooth edge [1]. In impactparameter space, denoting the transparency function by $\eta \equiv \eta\left(b, R_{\mathrm{TOT}}\left(W^{2}\right)\right)$, the representation (9) of the total cross section reads [1,2]

$$
\begin{aligned}
\sigma^{\mathrm{TOT}}\left(W^{2}\right) & =4 \pi \int d b b(1-\eta) \\
& =4 \pi \int d b b\left[(1-\eta)^{2}+\eta(1-\eta)\right],
\end{aligned}
$$

where, for the simplicity of the present presentation, a real part of the scattering amplitude is suppressed. The first and second parts on the right-hand side in (19) correspond to the first and second parts in (9) and (13).

The contribution to the total cross section that is due to the edge of constant width $t$ at half maximum is approximately bounded by values of the impact parameter $b$ in the range of

$$
R_{\mathrm{TOT}}\left(W^{2}\right)-t \leq b \leq R_{\mathrm{TOT}}\left(W^{2}\right)+t .
$$

The precise boundary depends on the exact shape of the transparency function. For impact parameters $b$ outside the range (20), in particular for values of $b$ approximately given by

$$
b \leq R_{\mathrm{TOT}}\left(W^{2}\right)-t \equiv b_{\mathrm{Max}}\left(W^{2}\right),
$$

the contribution of the edge to the total cross section in (9), (13), and (19) is vanishing. The total cross section obtained by integration over $b$ up to $b \leq b_{\text {Max }}\left(W^{2}\right)$ is approximately given by the black-disk value of

$$
\left.\sigma^{\mathrm{TOT}}\left(W^{2}\right)\right|_{b_{\text {Max }}\left(W^{2}\right)}=\left.2 \sigma^{\mathrm{EL}}\right|_{b_{\text {Max }}\left(W^{2}\right)}=2 \pi\left(R_{\mathrm{TOT}}\left(W^{2}\right)-t\right)^{2} .
$$

The fraction of the total cross section supplied by $\left.\sigma^{\mathrm{TOT}}\left(W^{2}\right)\right|_{b_{\mathrm{Max}}\left(W^{2}\right)}$ becomes

$$
\frac{\left.\sigma_{\mathrm{TOT}}\left(W^{2}\right)\right|_{b_{\mathrm{Max}}\left(W^{2}\right)}}{\sigma^{\mathrm{TOT}}\left(W^{2}\right)}=\left(1-\frac{t}{R_{\mathrm{TOT}}\left(W^{2}\right)}\right)^{2}=\left\{\begin{array}{l}
1 \text { for } R_{\mathrm{TOT}}\left(W^{2}\right) \gg t \\
0.063 \text { for } R_{\mathrm{TOT}}\left(W^{2}\right)=R_{\mathrm{TOT}}\left(W^{2}=(57 \mathrm{TeV})^{2}\right)=1.47 \mathrm{fm}, \\
0.028 \text { for } R_{\mathrm{TOT}}\left(W^{2}\right)=R_{\mathrm{TOT}}\left(W^{2}=(14 \mathrm{TeV})^{2}\right)=1.32 \mathrm{fm} .
\end{array}\right.
$$

Even at the highest presently accessible energies, the range in impact-parameter space of $b \leq b_{\operatorname{Max}}\left(W^{2}\right)$, where $\left.\sigma^{\mathrm{TOT}}\left(W^{2}\right)\right|_{b_{\operatorname{Max}}\left(W^{2}\right)}=\left.2 \sigma^{\mathrm{EL}}\right|_{b_{\operatorname{Max}}\left(W^{2}\right)}$, according to (21) and (23) is restricted to very small values of $b_{\mathrm{Max}} \cong 0.2$ to 0.4 fm. Compare also Refs. [2,8].
Figure $1^{1}$ shows eikonal fits of scattering amplitudes in impact-parameter space for various energies $W$ including

\footnotetext{
${ }^{1}$ The author thanks Phuoc Ha for preparing and providing Fig. 1 based on the eikonal fits from Ref. [2].
} 
the extrapolation to $W=50 \mathrm{TeV}$. The integrals over the impact parameter $b$ of the amplitudes shown in Fig. 1, upon multiplication by $4 \pi$, yield fits to the experimental data for $\sigma^{\mathrm{TOT}}\left(W^{2}\right)$ and $\sigma_{\text {Black }}^{\mathrm{TOT}}\left(W^{2}\right)=2 \sigma^{\mathrm{EL}}\left(W^{2}\right)$. The figure also shows a linearly rising theoretical black-disk amplitude. Upon integration, restricted by a chosen upper limit on $b$ and multiplication by $4 \pi$, one obtains the corresponding cross section of a black disk. The figure nicely displays the deviation between $\sigma^{\mathrm{TOT}}\left(W^{2}\right)=2 \sigma^{\mathrm{EL}}\left(W^{2}\right)=\sigma_{\text {Black }}^{\mathrm{TOT}}\left(W^{2}\right)$ and $\sigma^{\mathrm{TOT}}\left(W^{2}\right)=\sigma_{\mathrm{Black}}^{\mathrm{TOT}}\left(W^{2}\right)+\pi t R_{\mathrm{TOT}}\left(W^{2}\right)$ for impact parameters $b \geq b_{\text {Max }}\left(W^{2}\right)$ due to contributions from the edge. For $b>R_{\mathrm{TOT}}\left(W^{2}\right)$, the total cross section is dominantly due to the edge, and the edge is dominated by inelastic scattering. For $R_{\mathrm{TOT}}\left(W^{2}\right) \rightarrow \infty$, the relative contribution of the edge to the total cross section goes to zero, and $\sigma_{\text {Black }}^{\mathrm{TOT}}\left(W^{2}\right) / \sigma^{\mathrm{TOT}}\left(W^{2}\right) \rightarrow 1$. The huge energy required to approach this saturation limit of full blackness is inherently connected with both the fairly large value of the edge constant $t \simeq 1.1 \mathrm{fm}$ and the slow increase of $R_{\mathrm{TOT}}\left(W^{2}\right)$ with the energy $W$ that is due to the small coefficient $c_{2}$ of the $\ln ^{2}\left(W^{2}\right)$ term in the representation of the total cross section, $\sigma^{\mathrm{TOT}}\left(W^{2}\right)$. We note that a (theoretical) value of $t \rightarrow 0$, corresponding to a black-disk interaction at all energies, is not only incompatible with the experimental value of $t \simeq 1.1 \mathrm{fm}$, but it is also inconsistent with the original prediction and theoretical explanation of the $\ln ^{2}\left(W^{2}\right)$ dependence of the total cross section which relies [9] on an increase of the active interaction region, when increasing the energy.

The edge constant $t \simeq 1.1 \mathrm{fm}$ determines the partition of the total cross section $\sigma^{\mathrm{TOT}}\left(W^{2}\right)$ for given $W$ into an elastic and an inelastic part, and it quantifies the approach to the black-disk limit

$$
\lim _{W \rightarrow \infty} \frac{R_{\text {Black }}\left(W^{2}\right)}{R_{\mathrm{TOT}}\left(W^{2}\right)}=1
$$

as a function of the $p p$ energy $W$. It seems appropriate to explicitly elaborate on these interpretations of the work in (1).

\section{ACKNOWLEDGEMENTS}

This paper was inspired by a lecture given by Leo Stodolsky at the Max Planck Institut für Physik in München. The author thanks Leo Stodolsky for useful discussions and Phuoc Ha for providing Fig. 1.
[1] M. M. Block, L. Durand, F. Halzen, L. Stodolsky, and T. J. Weiler, Evidence for an energy-invariant "edge" in protonproton scattering at very high energies, Phys. Rev. D 91, 011501 (2015).

[2] M. M. Block, L. Durand, P. Ha, and F. Halzen, Eikonal fit to $p p$ and $\bar{p} p$ scattering and the edge in the scattering amplitude, Phys. Rev. D 92, 014030 (2015).

[3] M. M. Block, L. Durand, P. Ha, and F. Halzen, Comprehensive fits to high energy data for $\sigma, \gamma$ and $B$ and the asymptotic black-disk limit, Phys. Rev. D 92, 114021 (2015).

[4] L. Stodolsky, Behavior of very high energy hadronic cross sections, Mod. Phys. Lett. A 32, 1730028 (2017).

[5] M. M. Block and F. Halzen, Analyticity as a robust constraint on the total cross section at the CERN Large Hadron Collider, Phys. Rev. D 73, 054022 (2006).
[6] P. Abreu et al. (Pierre Auger Collaboration), Measurement of the Proton-Air Cross Section at $\sqrt{s}=57 \mathrm{TeV}$ with the Pierre Auger Observatory, Phys. Rev. Lett. 109, 062002 (2012)

[7] R. Abbasi et al. (HiRes Collaboration), An upper limit on the electron-neutrino flux from the hires detector, Astrophys. J. 684, 790 (2008).

[8] A. Alkin, E. Martynov, O. Kovalenko, and S. M. Troshin, Impact-parameter analysis of TOTEM data at the LHC: Black disk limit exceeded, Phys. Rev. D 89, 091501 (2014).

[9] W. Heisenberg, Vorträge über kosmische Strahlung (Springer, Berlin, 1953), p. 155; reprinted in Collected Works, Series B (Springer, Berlin, 1984), p. 498; The unified field theory of elementary particles: Some recent advances, Die Naturwiss. 61, 1 (1974); reprinted in Collected Works, Series B, (Springer, Berlin, 1984), p. 912. 